\title{
Improvement of self-crosslinking HXNBR by multi-walled carbon nanotubes in latex blending
}

\author{
Yue Dongmei ${ }^{1, \text { a }}$, Su Lin ${ }^{2, b}$ \\ Beijing North Third Ring Road on the 15th, Chaoyang District, Beijing University of Chemical \\ Technology \\ ayuedm@mail.buct.edu.cn, bsulinbuct@163.com
}

Keywords: self-crosslinking, multi-walled carbon nanotube, HXNBR

\begin{abstract}
The dispersed MWCNT was introduced into self-crosslinking HXNBR latex to get the compounded latex film. Mechanical, electrical and thermal properties of this composite were investigated in this article. Volume resistivity decreased significantly with the increase of the MWCNT loading. When the content of the MWCNT reached 3phr, the low percolation threshold value was obtained in this composite with the low dielectric loss. The composite showed good mechanical properties especially after aging because of the effect of self-crosslinking by heated.
\end{abstract}

\section{Introduction}

NBR is widely used in automotive, aerospace and oil-well industries due to its best oil resistance properties [1]. It is expected to hydrogenate the diene-based rubber to improve the oxidative stability of the rubber by replacing the residual double bonds in the butadiene segments. Hydrogenation processes are commonly carried out in solution with pressured hydrogen, rhodium, and transition-mental catalysts. Wideman [2] and Belt [3] found a method for the direct conversion of NBR latex to HNBR latex without the use of high-pressure hydrogen gas, precious metal catalysts, organic solvent and special facilities. However, the gel existed when the rubber got dried. Yue dongmei [4] applied the crosslink of HXNBR latex to form a kind of film and got good mechanical properties without any vulcanizing agent.

The HXNBR is an insulating polymer which is not electrically conductive. It is necessary to integrate conductive filler into HXNBR matrix to make the HXNBR conductive enough for practical use. Because of the excellent mechanical, thermal and electrical properties [5]-[6], carbon nanotubes, are currently attractive among reinforcing filler. A lot of researches have been done to investigate the properties of rubber with the MWCNT as the filler [7]-[8]. In this article, our aim is to use the properties of self-crosslinking in HXNBR, prepare the blends with various MWCNT loading by mixing HXNBR and MWCNT and investigate the mechanical, thermal and electrical properties.

\section{Experiment}

\section{Materials}

Carboxylated Nitrile Butadiene Rubber latex was purchased from Zeon Company (Japan). MWCNT was obtained from Chengdu Institute of Organic Chemistry. Hydrazine hydrate, aqueous solution of hydrogen peroxide, Sodium dodecyl benzenesulfonate and boric acid were obtained from Beijing Chemical Plant(China).

\section{Hydrogenation of XNBR Latex}

XNBRL, hydrazine hydrate and distilled water was added into a 500ml three necked flask with stirring. The mixture was heated to $45{ }^{\circ} \mathrm{C}$ and stirred for $30 \mathrm{~min}$. The solution of boric acid and hydrogen peroxide was introduced drop by drop.

\section{Preparation of the compound film}

Sonicating an aqueous suspension of nanotube with surfactant SDBS for about $15 \mathrm{~min}$ and the carbon nanotube dispersions were prepared. Then, the dispersion of carbon nanotube was introduced into the HXNBRL drop by drop with magnetic stirring. The compounded latex was cast 
on the former with coagulants and dried in air to form wet film. After the wet film forming, the wet film was dipped into the distilled water in $40^{\circ} \mathrm{C}$ for $12 \mathrm{~h}$ then dried in $70^{\circ} \mathrm{C}$ for $48 \mathrm{~h}$.

\section{Characterization}

The converse of double bonds to single bonds in the NBR latex was determined by Fourier transform infrared spectrometry. Tensile tests of latex film specimens were carried out on a CMT4101 testing machine. The surface morphology of section was investigated using a Britain Cambridge scanning electron microscope.

The volume resistivity was measured using high resistance meter. The dielectric properties of the samples were measured using impedance analyzer.

\section{Results and discussion}

\section{Structure Aspects of HXNBR Characterization}

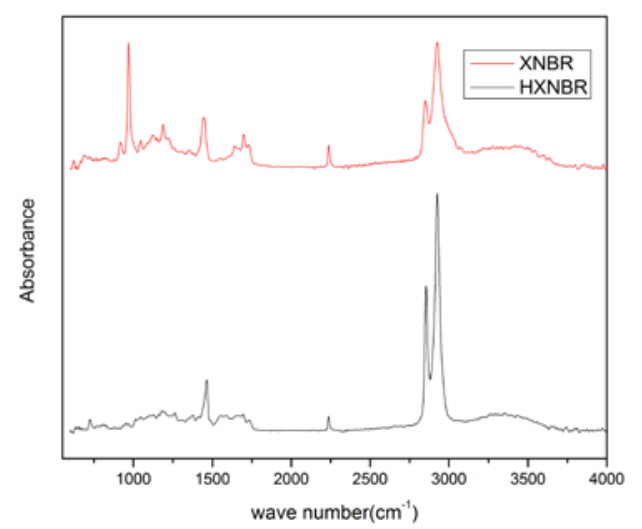

Fig1. ATR-FTIR spectra of (a) HXNBR, (b) XNBR

In figure1, Spectral intensity in HXNBR are detected at 2237, 967, 917, and 740 $\mathrm{cm}^{-1}$ compared to XNBR, corresponding to C-N stretching, 1,4 trans butadiene unit stretching, 1,2 vinyl stretching, and 1,4 cis butadiene unit stretching, respectively. The peak of butadiene units decrease in height as the extent of $\mathrm{C}=\mathrm{C}$ unsaturation decrease and a new peak at $722 \mathrm{~cm}_{-1}$ as a result of $-\mathrm{CH}_{2}$ - rocking vibrations is observed. There is no change in the intensity of the absorption peak at $2237 \mathrm{~cm}-1$, reflecting the stability of the nitrile groups towards diimide reduction.

\section{Mechanical properties}

The stress-strain curves were presented in figure2, it can be seen that the tensile strength increased with the increase of the content of MWCNT and reached a peak value when the content of MWCNT was 1phr and then decreased, the elongations at break showed the same trend. SDBS strengthened the combination between HXNBR and MWCNT and weakened the ability of agglomeration and entanglement of MWCNT. So the MWCNT has a reinforcing function to matrix of HXNBR.

As can be seen in figure3, tensile strength all increased after aging. The difference between HXNBR and other rubber is that the chain got further crosslinking after heated without any vulcanization reagents. 


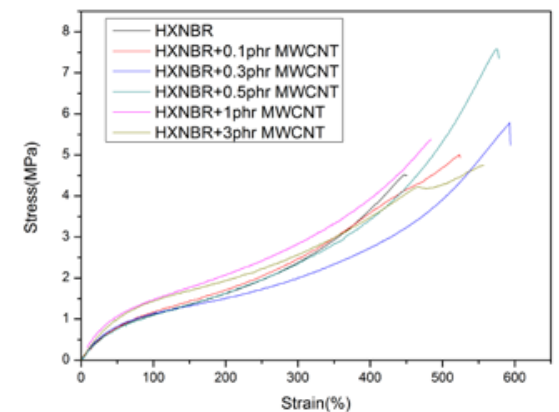

(1)

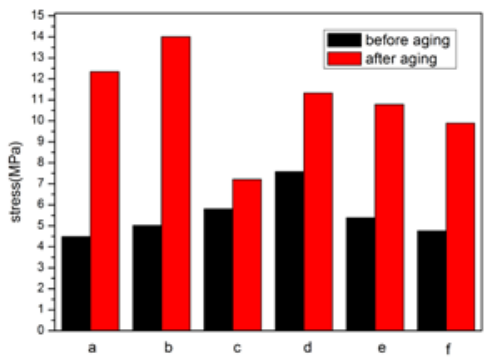

(2)

Fig2. (1) Tensile strain-stress curves of compounded latex film. (2)Comparison of tensile strength before and after aging: (a) HXNBR, (b) 0.1phr MWCNT+HXNBR, (c) 0.3phr MWCNT+HXNBR

(d) 0.5phr MWCNT+HXNBR (e) 1phr MWCNT+HXNBR (f) 3phr MWCNT+HXNBR.

\section{SEM analysis}

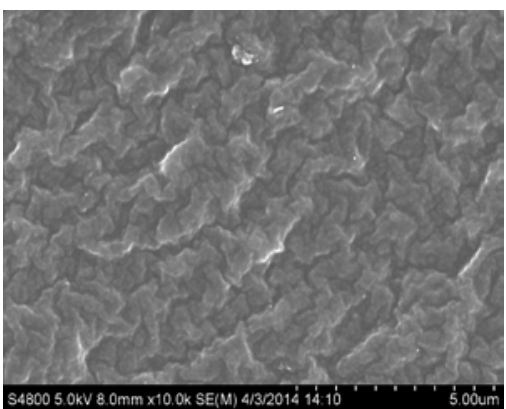

(a)

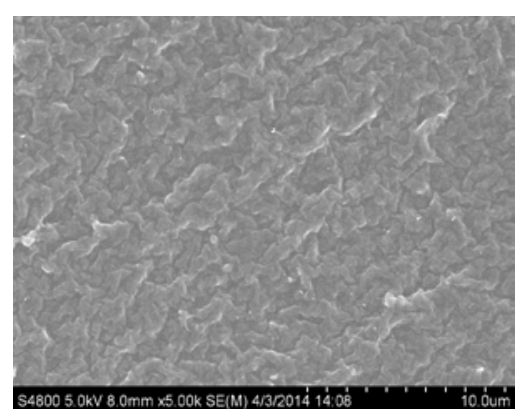

(b)

Fig3. SEM images of section of HXNBR with 0.5 phr MWCNT: (a) $\times 10 \mathrm{k}$, (b) $\times 5 \mathrm{k}$

SEM images of section of HXNBR with 0.5phr MWCNT is shown in Figure4, it can be seen from the SEM of the section of sample that white dots and lines were the MWCNT. MWCNT dispersed evenly in HXNBR basic bodies, resulting in a reinforcing effect on the latex film. Furthermore, because we mix HXNBR and MWCNT in latex stage instead of mechanically mixing like others [8], there is no broken MWCNT.

\section{Electrical properties}

Volume resistivity is shown in table2, it can be seen that the Volume resistivity dropped significantly from $1.4 \times 10^{10}$ (HXNBR) to $5.7 \times 10^{6}$ (HXNBR+3phr MWCNT), the abrupt decrease results from the formation of a continuous CNT network in the HXNBR matrix.

Figure5. shows the dielectric constant (E') and dielectric loss (E”). In the unfilled sample the dielectric constant has low value and behaved almost independent of frequency. With the increase of the MWCNT loading, the dielectric constant and dielectric loss increase too. The increase could be attributed to the increase in the polarity of the bond which leads to an increase in the interfacial polarization. A sharp increase in dielectric constant is observed with the presence of 3phr MWCNT, suggesting that the low percolation threshold value is obtained by distribution of MWCNT.

Table1. Volume resistivity of HXNBR with different MWCNT loading

\begin{tabular}{cc}
\hline Content of MWCNT (phr) & Volume resistivity $(\Omega \cdot \mathrm{cm})$ \\
\hline 0 & $1.4 \times 10^{10}$ \\
0.1 & $1.3 \times 10^{9}$ \\
0.3 & $8.6 \times 10^{8}$ \\
0.5 & $4.4 \times 10^{8}$ \\
1 & $4.4 \times 10^{8}$ \\
3 & $5.7 \times 10^{6}$ \\
\hline
\end{tabular}



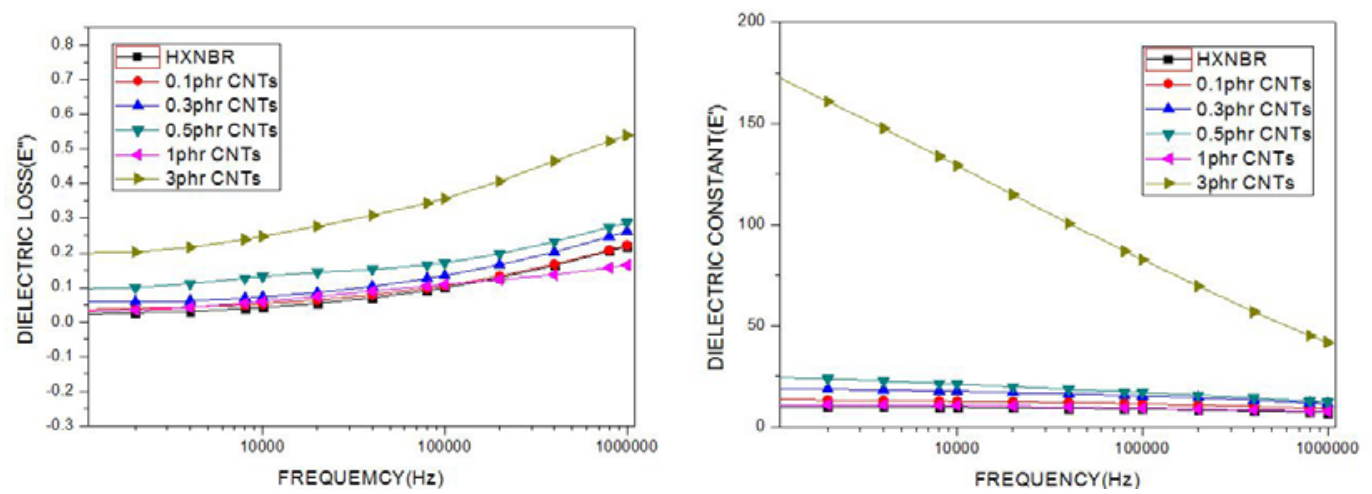

Fig4. Dielectric constant and dielectric loss of HXNBR with different MWCNT loading

\section{Conclusion}

SDBS and latex blending gave the MWCNT a uniform dispersion in HXNBR matrix. This can be seen from the SEM images of composites. The tensile strength, elongation at break went up to an optimum and then decreased. The tensile strength were all higher than before after aging because of the self-crosslinking ability. The volume resistivity decreased with the increase of the MWCNT loading and a low percolation threshold was obtained at around 3phr MWCNT with the loss dielectric loss.

\section{References}

[1] Kingender, R. C.; Oyama, M.; Saito, Y. Rubber World 1990, 202(3), 628.

[2] Wideman, L. G. U.S. Pat. 4,452,950, 1984 (to The Goodyear Tire \& Rubber Co.).

[3] Belt, J. W.; Vermeulen, J. A. A.; Ko stermann, M. WO 00/09576,2000.

[4] Yang Han; Lixin Mao; Hongwei Meng; Liqun Zhang; Dongmei Yue. J.APPL.POLUM.SCI DOI: 10.1002/app.39865

[5] Evgeniy E. Tkalya , Marcos Ghislandi , Gijsbertus de With , Cor E. Koning. Current Opinion in Colloid \& Interface Science 17 (2012) 225-232

[6] X.L. Xie; Y.M. Mai; X. Ping. Master. Sci. Eng 49 (4) (2005) 89.

[7] K. Preetha Nair, Paulbert Thomas , Rani Joseph. Materials and Dsigin 41(2012) 23-30

[8] Songmin Shang, Lu Gan , Marcus Chun-wah Yuen, Shou-xiang Jiang, Nicy Mei Luo. Composite Part A 66(2014)135-141 